\title{
Proteomic response of Moniliophthora perniciosa exposed to pathogenesis-related protein-10 from Theobroma cacao
}

\author{
F.A.C. Silva ${ }^{1}$, C.P. Pirovani ${ }^{1}$, S. Menezes ${ }^{1}$, C. Pungartnik ${ }^{2}$, A.S. Santiago ${ }^{1}$, \\ M.G.C. Costa ${ }^{1}$, F. Micheli ${ }^{3}$ and A.S. Gesteira ${ }^{4}$ \\ ${ }^{1}$ Laboratório de Biologia Molecular, Centro de Biotecnologia e Genética, \\ Universidade Estadual de Santa Cruz, Ilhéus, BA, Brasil \\ ${ }^{2}$ Laboratório de Biologia de Fungos, Centro de Biotecnologia e Genética, \\ Universidade Estadual de Santa Cruz, Ilhéus, BA, Brasil \\ ${ }^{3}$ Genetic Improvement and Adaptation of Mediterranean and Tropical Plants, \\ Joint Research Unit, Agricultural Research for Development, \\ Montpellier, France \\ ${ }^{4}$ Embrapa Mandioca e Fruticultura, Departamento de Biologia Molecular, \\ Cruz das Almas, BA, Brasil
}

Corresponding author: A.S. Gesteira

E-mail: Abelmon.Gesteira@embrapa.br

Genet. Mol. Res. 12 (4): 4855-4868 (2013)

Received July 17, 2013

Accepted September 18, 2013

Published October 22, 2013

DOI http://dx.doi.org/10.4238/2013.October.22.5

\begin{abstract}
TcPR-10, a member of the pathogenesis-related protein 10 family, was identified in EST library of interactions between Theobroma cacao and Moniliophthora perniciosa. TcPR-10 has been shown to have antifungal and ribonuclease activities in vitro. This study aimed to identify proteins that are differentially expressed in $M$. perniciosa in response to TcPR-10 through a proteomic analysis. The fungal hyphae were subjected to one of four treatments: control treatment or $30-, 60-$ or $120-\mathrm{min}$ treatment with the TcPR-10 protein. Twodimensional maps revealed 191 differentially expressed proteins, 55 of which were identified by mass spectrometry. The proteins identified in all treatments were divided into the following classes: cell metabolism,
\end{abstract}


stress response, zinc binding, phosphorylation mechanism, transport, autophagy, DNA repair, and oxidoreductases. The predominant class was stress-response proteins $(29 \%)$, such as heat shock proteins; these proteins exhibited the highest expression levels relative to the control treatment and are known to trigger defense mechanisms against cytotoxic drugs as well as TcPR-10. Oxidoreductases (25\%) were overexpressed in the control and in 30-min treatments but exhibited reduced expression at $120 \mathrm{~min}$. These proteins are involved in the repair of damage caused by oxidative stress due to the contact with TcPR10. Consistent with the antifungal activity of TcPR-10, several proteins identified were related to detoxification, autophagy or were involved in mechanisms for maintaining fungal homeostasis, such as ergosterol biosynthesis. These results show that the sensitivity of the fungus to TcPR-10 involves several biochemical routes, clarifying the possible modes of action of this antifungal protein.

Key words: Pathogenesis-related protein 10; Oxidoreductases; Differentially expressed proteins; Stress-response proteins

\section{INTRODUCTION}

Moniliophthora perniciosa (Basidiomycota, Agaricales, Marasmiaceae) is the causal agent of witches' broom disease (Aime and Phillips-Mora, 2005) in the cacao tree (Theobroma cacao L.). This disease is considered the most important cause of loss of cacao production in the South America and Caribbean, with important social, economical, and ecological impacts on the affected regions (Kilaru and Hasenstein, 2005; Scarpari et al., 2005). The life cycle of M. perniciosa is hemibiotrophic and is divided into i) biotrophic (parasitic) phase, characterized by monokaryotic hyphae that induce hyperplasia and hypertrophy of the plant tissue, loss of apical dominance, axillary shoot proliferation, and abnormal stems (green broom), and ii) necrotrophic (saprophytic) phase, characterized by dikaryotic hyphae containing clamp connections that correspond, at the plant level, to the necrosis and death of infected tissues distant from the primary infection site (dry broom). Basidiocarp production and spore formation occur on the surface of the necrotic tissues of the infected plant (Aime and Phillips-Mora, 2005).

The major damage caused by witches' broom disease has motivated several genomic and proteomic studies for a better understanding of the physiology of this pathogen and its mechanism of attack on cocoa. Gesteira et al. (2007) constructed cDNA libraries from two cacao cultivars, one resistant and one susceptible to M. perniciosa, and identified the TcPR-10 gene (pathogenesis-related protein 10 of T. cacao). TcPR-10 is a member of a family of acidic proteins (PR-10) that are found in some gymnosperms and angiosperms and that are responsive to intracellular defense processes (Islam et al., 2009; Xie et al., 2010). Members of the PR10 family have a p-loop motif, a highly conserved glycine-rich motif (GXGGXGXXK; 4755 amino acids) that is involved in ribonuclease activity (Lytle et al., 2009). Another feature of the PR10 family is the presence of phosphorylation sites that are characteristic of cAMPdependent kinases (Bantignies et al., 2000). The presence of three common phosphorylation sites in PR-10 proteins suggests a general or specific RNase effect against exogenous RNA (Park et al., 2004). Recent reports identified DNAse activity in PR-10 proteins in rice (Kim 
et al., 2011) and grape (He et al., 2013), suggesting that this protein may act as a nuclease in programmed cell death.

In 2009, Pungartnik et al. reported the ribonuclease and antifungal activity of the heterologous protein TcPR-10 against M. perniciosa and Saccharomyces cerevisiae. The authors also showed an in vitro antifungal activity of TcPR-10 leading to the inhibition of $M$. perniciosa growth. Additionally, Menezes et al. (2012) showed that the allergenic potential of TcPR-10 may be diminished or abolished by the introduction of specific point mutations into the protein without disrupting its RNase activity. They showed that TcPR-10 has a strong RNAse activity against $M$. perniciosa RNA, and that this response is both concentration- and time-dependent. Several PR-10 protein family members have been reported to hydrolyze (Liu et al., 2005; Yan et al., 2008) and this activity can be crucial during the attack of a pathogen, because it activates apoptotic processes in the pathogen to limit the invasion.

The ribonuclease properties and antifungal activity of PR-10 molecules are widely described in the literature. Park et al. (2004) identified CaPR-10, a protein expressed in the hot pepper (Capsicum annuum) that is known to inhibit the hyphal extension of Phytophthora capsici, possibly by inhibiting its translation activity. Pinto and Ricardo (1995) identified a group of inducible low molecular weight acidic proteins with $\mathrm{N}$-terminal amino acid sequences similar to those of the PR-10 proteins in leaves from Lupinus albus. These proteins, PR-p16.5, PR-p16.5b, and PR-p16.5c, are induced upon infection by Colletotrichum gloeosporioides. Flores et al. (2002) described an antifungal PR-10 group protein, the 18-kDa storage protein ocatin in the oca (Oxalis tuberosa), an Andean tuber. In vitro, this protein inhibits the growth of several phytopathogenic fungi, including Fusarium oxysporum and Rhizoctonia solani.

The use of proteomic tools to assess the effects of antifungal agents has been successfully applied to provide a comprehensive examination of the changes occurring in the pathogen. Cagas et al. (2011) investigated the changes in the Aspergillus fumigatus proteome in response to the stress generated by coumarin, a known antifungal drug, and the possible mechanism of action by which the compound produces lethal effects against the fungus.

In this study, we used proteomic analyses to identify proteins that are differentially expressed in M. perniciosa hyphae treated with TcPR-10, or not treated (control), to elucidate the mechanisms that are directly or concurrently involved in the response of $M$. perniciosa to the antifungal compound TcPR-10. To our knowledge, this is the first study describing and analyzing proteins expressed by M. perniciosa in response to PR-10 from T. cacao.

\section{MATERIAL AND METHODS}

\section{Obtaining the TcPR-10 protein}

The TcPR-10 gene (accession No. ES439858) was isolated from the T. cacao x M. perniciosa interaction cDNA library described by Gesteira et al. (2007). The gene was cloned into the expression vector pET28a, and the TcPR-10 recombinant protein was expressed in Escherichia coli BL21 (DE), as previously described by Pungartnik et al. (2009) and Menezes et al. (2012). A stock solution of recombinant TcPR-10 protein was prepared at a concentration of $30 \mathrm{mg} / \mathrm{mL}$.

\section{M. perniciosa growth conditions}

M. perniciosa strain ALF553 cultures were grown as described by Filho et al. (2006). 
Dikaryotic cultures were grown in CPD ( $2 \%$ glucose, $2 \%$ peptone) in liquid medium without agitation at $25^{\circ} \mathrm{C}$ for 5 to 7 days. M. perniciosa was obtained from the fungal collection of UESC, originally kept at Comissão Executiva do Plano da Lavoura Cacaueira.

\section{Exposure of the fungus M. perniciosa to TcPR-10}

The M. perniciosa hyphae were incubated with the recombinant TcPR-10 protein (final concentration of $3 \mu \mathrm{g} / \mathrm{mL}$ ) for 30,60, or $120 \mathrm{~min}$ as described by Filho et al. (2006). A culture that was not exposed to the protein was used as a control. After harvesting, the fungal hyphae were frozen in liquid $\mathrm{N}_{2}$ and stored at $-80^{\circ} \mathrm{C}$. Three independent experiments were performed for each incubation time.

\section{M. perniciosa protein extraction and quantification}

Protein was extracted from M. perniciosa according to Pirovani et al. (2008). Protein extracts were subjected to a second cleaning method for total protein purification as described by Meyer et al. (1988). The proteins were then quantified using the 2-D Quant kit according to manufacturer instructions (GE Healthcare, Milwaukee, WI, USA) and using bovine serum albumin as standard.

\section{2-D gel electrophoresis}

Isoelectric focusing was performed with $350 \mathrm{mg}$ protein in a final volume of $250 \mu \mathrm{L}$ rehydration solution (7 M urea, $2 \mathrm{M}$ thiourea, 1\% CHAPS, $100 \mathrm{mM}$ DTT, 0.5\% IPG buffer, $\mathrm{pH} 3-10$, containing a pinch of bromophenol blue). Samples were applied to $13-\mathrm{cm}$ IPG strips with a linear $\mathrm{pH}$ gradient from 3 to 10 (Amersham Biosciences, Immobiline DryStrip ${ }^{\mathrm{TM}}$ ). The strips were treated with equilibration buffer (6 M urea, $2 \% \mathrm{SDS}, 30 \%$ glycerol, $50 \mathrm{mM}$ Tris$\mathrm{HCl}, \mathrm{pH} 8.8$ ) containing 1\% DTT (dithiothreitol) and equilibration buffer containing $2.5 \%$ iodoacetamide. The second dimension of the electrophoresis was performed with a Hoefer SE 600 vertical Ruby (GE Healthcare) electrophoresis system at $15^{\circ} \mathrm{C}$ under $80 \mathrm{~V} / 200 \mathrm{~mA}$. The gels were fixed in a solution containing $40 \%$ ethanol and $10 \%$ acetic acid for $1 \mathrm{~h}$ and then stained using Coomassie brilliant blue $\mathrm{G}$ according to manufacturer instructions (Sigma).

\section{2-D gel image analysis}

Three 2-D gels were obtained for each incubation time, and the most representative gel was chosen as the reference for the analysis. The images of the 2-D gels were produced by the ImageScanner II (GE Healthcare) at a resolution of $600 \mathrm{dpi}$, and the digitized images were analyzed using the Image Master 2-D Platinum v.7.0 program (GE Healthcare) for spot detection, quantification and matching. After automated detection and matching, manual editing was performed for individual spots.

Gel groups were established according to the experimental design, and spot-normalized volume was used to select statistically significant different (fold-change, ANOVA, falsediscovery rate) spots between the treatments analyzed in the experiment. The spot values were normalized using the following formula: intensity of each spot/total intensity, in which the total intensity refers to the sum of all spots belonging to the same gel. Internal molecular markers were used to determine the experimental isoelectric point (pI) and molecular weight for each protein of interest. 
Significant differences between incubation times were determined for each spot. Oneway ANOVA was applied to compare the percentage volume values of matched spots. A spot was considered to be differentially expressed if it exhibited a fold-change higher than 1.2. The ratio of the accumulation of proteins was quantified by Nano LC-ESI-Q-TOF performed with ImageMaster 7.0/GE, using the control treatment as reference.

\section{Preparation of spots for mass spectrometry (MS)/MS}

The differentially expressed spots were manually excised from the 2-D gel, and the gel pieces were destained in $\mathrm{NH}_{4} \mathrm{HCO}_{3}$ containing $50 \%$ acetonitrile $(\mathrm{ACN})$. Gel fragments were dehydrated in ACN and dried in a SpeedVac (Eppendorf). Next, a $25-\mathrm{ng} / \mu \mathrm{L}$ trypsin solution (Gold Promega) was added to the sample, which was then incubated at $4^{\circ} \mathrm{C}$ for $10 \mathrm{~min}$ to allow residual solution to be absorbed by the gel fragments. $\mathrm{NH}_{4} \mathrm{HCO}_{3}$ was then added to cover the pieces, and the samples were incubated at $37^{\circ} \mathrm{C}$ for $16 \mathrm{~h}$. The supernatant was collected and transferred to a new tube. The gel fragments were washed twice with $50 \%(\mathrm{v} / \mathrm{v}) \mathrm{ACN}$ and $0.1 \%$ $(\mathrm{v} / \mathrm{v})$ trifluoroacetic acid, and the supernatant was collected. The supernatants obtained in the last two steps were pooled and concentrated under vacuum to a volume of $20 \mu \mathrm{L}$.

\section{MS analysis}

MS was performed using NanoLC-ESI-Q-TOF Micro ${ }^{\mathrm{TM}}$ (Micromass, Waters, Milford, MA, USA). The peptides were separated by nano-chromatography using a reversed phase column (nanoAcquity UPLC - Waters) coupled to a mass spectrometer NanoLC-ESI-Q-TOF Micro $^{\mathrm{TM}}$ (Micromass, Waters). Initially, the peptides were desalted using a Symmetry C18 precolumn (Waters) $(5 \mu \mathrm{m}, 180 \mu \mathrm{m}$ in internal diameter x $20 \mathrm{~mm}$ in length) and an analytical column $(1.7 \mu \mathrm{m} \mathrm{BEH} 300, \mathrm{C} 18100 \mu \mathrm{m} \times 100 \mathrm{~mm})$. The flow rate was $0.6 \mu \mathrm{L} / \mathrm{min}$ with $50 \mathrm{~min} / \mathrm{run}$, and $4 \mu \mathrm{L}$ of each sample was injected. The peptides were separated using a gradient of $1 \% \mathrm{ACN}$ and then ionized in a capillary at $3000 \mathrm{~V}$, fragmented in positive ion mode, with a cut-off of a relative intensity of at least 10 counts; the three most intense ions (scans) were analyzed with collision energies ranging between 20 and $95 \mathrm{eV}$ according to the mass/load (mz) of peptide.

The MS data analysis was performed using the Masslynx 4.0 software (WatersMicromass, Manchester, UK). Protein identification was performed using a Mascot server (Version 2.1.04, Matrix Science) and the Magnaporthe grisea (http://www.broadinstitute.org/ annotation/fungi/magnaporthe/) database. The parameters for the MS data analysis were the following: i) only tryptic peptides with up to one missed cleavage site; ii) carbamidomethyl cysteine as a fixed modification; and iii) oxidized methionine as a variable modification.

\section{RESULTS AND DISCUSSION}

In the present study, 2-D gel analysis and MS were used to elucidate the adaptive responses of M. perniciosa to the antifungal agent TcPR-10. Proteins with different abundances at different exposure times were identified. The 2-D maps displayed a profile of proteins with molecular weights ranging from 3 to $201 \mathrm{kDa}$ and $\mathrm{pI}$ values from 3 to 10 . We observed that, in the control treatment, $69 \%$ of spots had a pI value between 3 and 7 , and $31 \%$ had a pI value between 7 and 10; in the $30-$ min treatment gel, $61 \%$ of spots had a pI value between 3 and 7 , and $39 \%$ had a pI value between 7 and 10; in the 60 -min treatment gel, $62 \%$ of spots had a pI value between 3 
and 7 , and $38 \%$ had a $\mathrm{pI}$ value between 7 and 10; and in the 120-min treatment gel, 50\% of spots had a pI value between 3 and 7, and 50\% had a pI value between 7 and 10 (Figure 1).

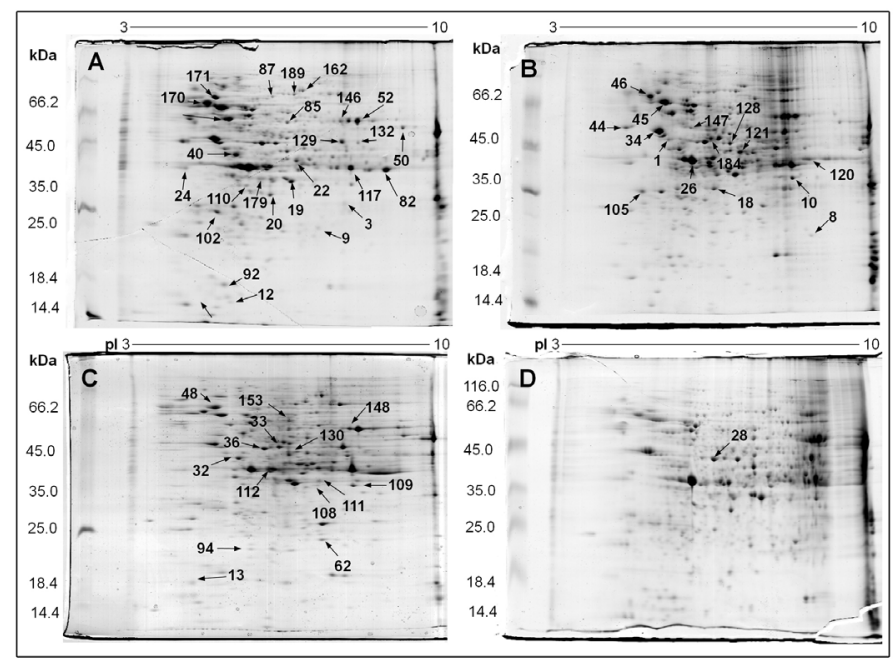

Figure 1. Protein profile on a 2-DE gel of the fungus Moniliophthora perniciosa treated with the antifungal protein TcPR-10 (3 $\mu \mathrm{g} / \mathrm{mL}$ ) obtained from cacao. A. Control (without TcPR-10); B. $30 \mathrm{~min}$; C. $60 \mathrm{~min}$; D. $120 \mathrm{~min}$ after exposure to TcPR-10. The gels were stained with colloidal Coomassie blue G-250 solution. Arrows indicate spots excised from the gel for differentially expressed proteins identified by mass spectrometry. $\mathrm{pI}=$ isoelectric point.

The number of protein spots from each treatment, the number of differentially expressed proteins between treatments, and the exclusive proteins are presented in a Venn diagram (Figure 2). A total of 461 differentially expressed proteins were analyzed by MS, but only 55 were identified (Tables 1 and 2; Figure 1).

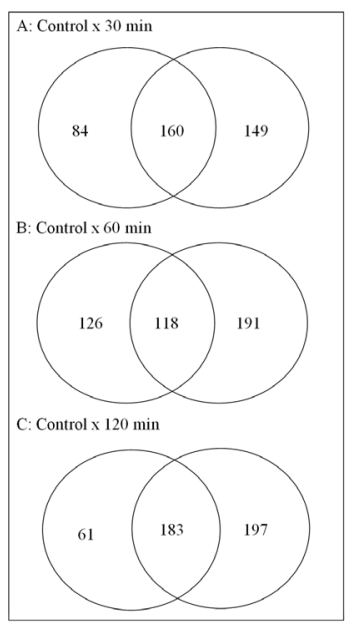

Figure 2. Venn diagram of the proteomic analysis of the fungus Moniliophthora perniciosa after exposure to TcPR$10(3 \mu \mathrm{g} / \mathrm{mL})$ or the control treatment (without TcPR-10) for 30, 60 and $120 \mathrm{~min}$. The number of differentially expressed spots was determined using the 2-D gel image analysis software. The statistical analyses of the matched proteins and spots were performed using ANOVA. 


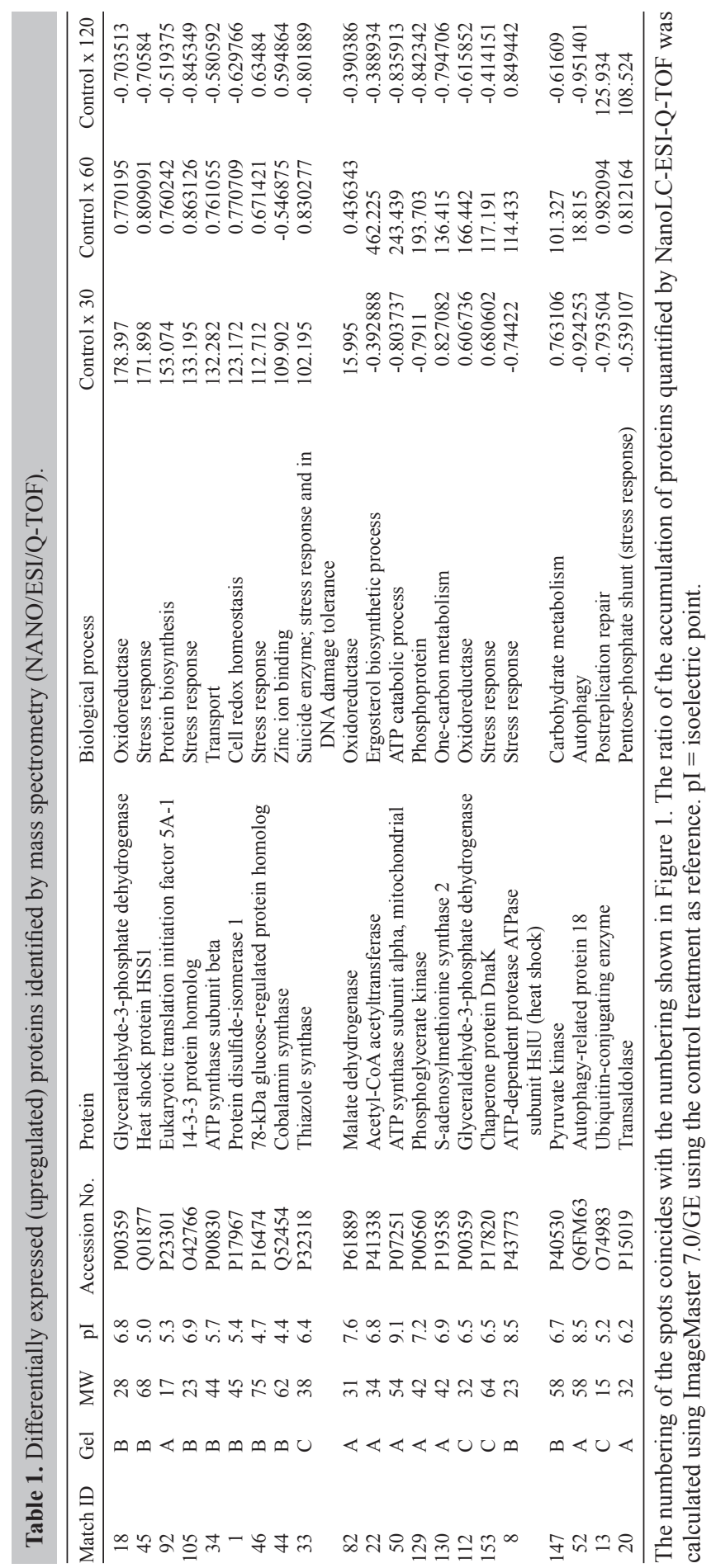


F.A.C. Silva et al.

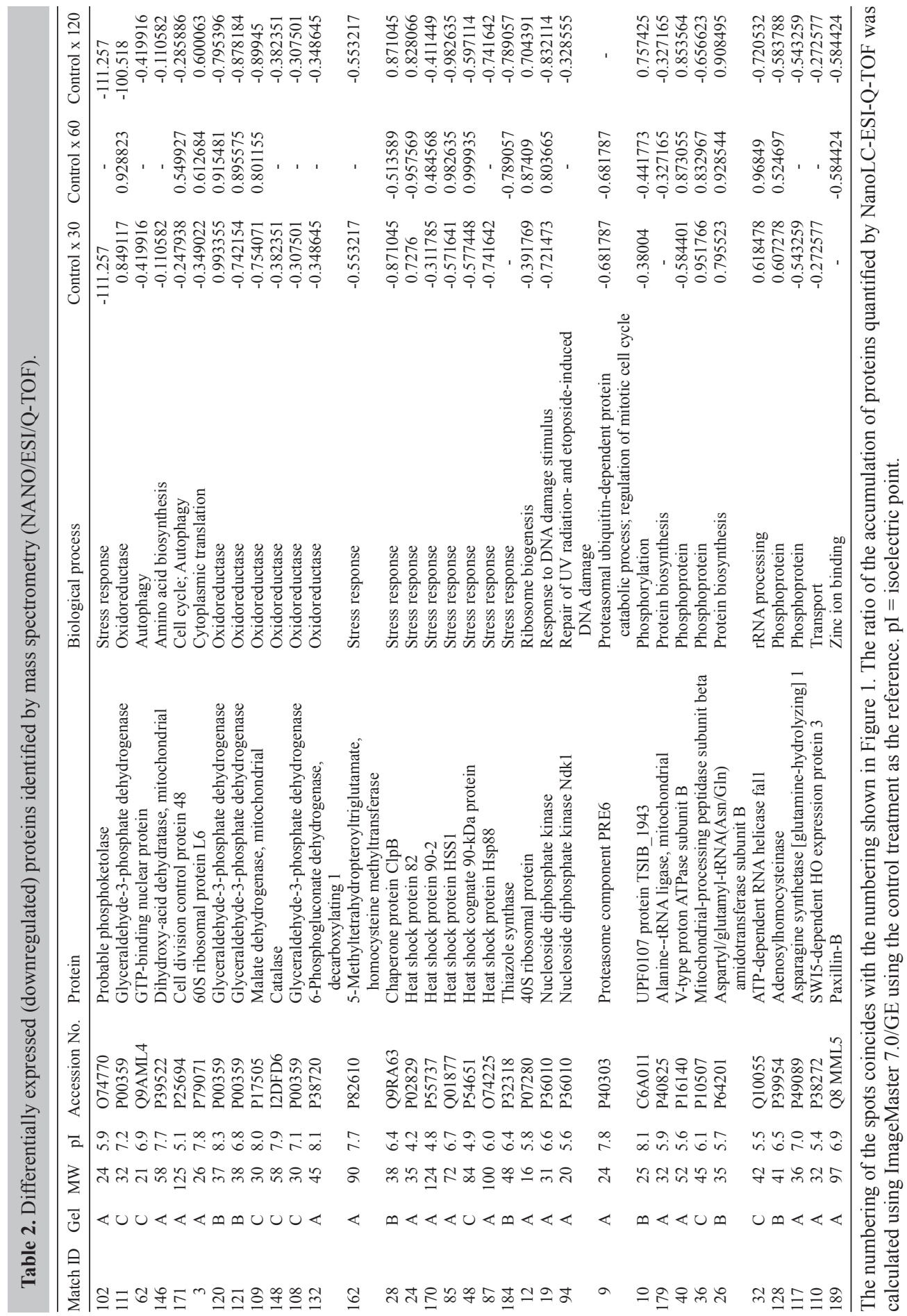




\section{0-min stress}

At $30 \mathrm{~min}$, certain proteins were overexpressed over 100 times compared to the control treatment, e.g., eukaryotic translation initiation factor 5A-1 (related to protein biosynthesis), protein disulfide-isomerase 1 (cell redox homeostasis), and thiazole synthase suicide enzyme (stress response and DNA damage tolerance).

Several PR-10 protein family members have been reported to hydrolyze RNA and this ribonuclease activity, when perceived by the cell, induces the synthesis of proteins required for the reduction of the associated damage. Thus, ATP synthase is mobilized to meet the ATP requirements of the cell, and cobalamin synthase is increased to provide a substrate for Krebs cycle activities (ATP synthase subunit beta, for transport; and cobalamin synthase, for zinc ion binding). Cobalamin, also known as vitamin B12, is used by mammalian cells in methylcobalamin and adenosylcobalamin, cofactors that are required for the methylation of homocysteine to methionine in the cytosol and for the conversion of methylmalonyl coenzyme A to succinyl coenzyme A in mitochondria, respectively (Zhao et al., 2013).

Under stress, the metabolism of the fungus $M$. perniciosa is strictly aerobic. Therefore, the preferred route of energy use is aerobic, using ergosterol derivatives that can be easily mobilized to the membrane (Huang and Tang, 2007). Glyceraldehyde-3-phosphate is oxidized to dihydroxyacetone phosphate by glyceraldehyde-3-phosphate dehydrogenase, and this reaction uses ATP and produces NADH (used directly in oxidative phosphorylation). Glyceraldehyde-3-phosphate dehydrogenase (spots 18, 102, 108, 111, 112, 121) is a key glycolytic enzyme that exists primarily in the cytoplasm (Hara et al., 2005), but when in the nucleus, it plays a role in gene transcription, DNA replication, DNA repair, and nuclear RNA export. Glycerol is a substrate for glycolysis, but during the aerobic production of ATP, it is considered a non-fermentable carbon source (Nevoigt and Stahl, 1997). The enzyme 6-phosphogluconate dehydrogenase (6PGDH; decarboxylating) (spot 132) catalyzes the rate-limiting NADPH-producing step in the pentose phosphate pathway. 6PGDH plays an important role in the tolerance against oxidative stress (Izawa et al., 1998).

We noted that once the cells perceive the damage, primarily involving oxidative stress, antioxidant defenses are activated (stress response proteins, heat shock protein HSS1, 78-kDa glucose-regulated protein homolog, and 14-3-3 protein homolog). One striking feature of the 14-3-3 proteins is their ability to bind a multitude of functionally diverse signaling proteins, including kinases, phosphatases, and transmembrane receptors. This variety of interacting proteins allows 14-3-3 to play important roles in a wide range of vital regulatory processes, such as mitogenic signal transduction, apoptosis, and cell cycle control (Fu et al., 2000).

Heat shock proteins (spots 24, 45, 48, 85, 87, 153, and 170) are molecular chaperones that are found in all organisms and are natural defenses against various types of stress, e.g., oxidative stress caused by drugs or environmental changes (Wandinger et al., 2008).

Oxidoreductases were observed as well (spots 18, 82, 108, 109, 111, 112, 120, 121, 132, and 148; Figure 1; Table 1). Oxidoreductases are involved in antioxidant defense, the maintenance of intracellular redox balance and the repair of damage caused by oxidative stress $(\mathrm{Xu}, 2005)$. Mur et al. (2004) observed that PR-10 gene expression was influenced by the presence of reactive oxygen species (ROS) and observed an overexpression of AoPR-10-GUS in transgenic Arabidopsis thaliana when the plants were exposed to salicylic acid, an important inducer of ROS, or in the presence of Pseudomonas syringae virulence factor. These 
oxidoreductases may be expressed by the fungus to withstand the oxidative damage caused by TcPR-10, reducing the expression of TcPR-10 at $120 \mathrm{~min}$. These results indicate that ROS have a potential negative influence on TcPR-10 expression. ROS, which are highly potent oxidants, are produced during normal metabolic processes or by an unfavorable environment. Within the cell, ROS react with macromolecules, including lipids, carbohydrates, DNA and proteins, to trigger molecular damage, such as DNA mutations, lipid peroxidation, and protein oxidation, eventually leading to cell death and the progressive aging of the organism (Heller and Tudzynski, 2011). Eukaryotic cells contain mechanisms to respond to and protect against stress conditions, including neutralization of the stress, pausing of the cell cycle, alterations in translation, and the repair of damage to apoptotic or necrotic pathways (Thompson et al., 2008). The stress induced in fungal cells by drugs such as TcPR-10 triggers some defense mechanisms against oxidative stress, of which antioxidants are relevant endpoints.

Also at $30 \mathrm{~min}$, we observed the suppression of the expression of phosphoketolase, a probable stress response protein (reduced $>100$-fold). The two substrates of this enzyme are D-xylulose 5-phosphate and phosphate, and its three products are acetyl phosphate, Dglyceraldehyde-3-phosphate and $\mathrm{H}_{2} \mathrm{O}$ (Ronne, 1995).

\section{0-min stress}

The entire synthesis process must proceed within 30 min with an intense production of ATP. The following proteins were induced ( $>100$-fold): glyceraldehyde-3-phosphate dehydrogenase (oxidoreductase), ATP-dependent protease ATPase subunit HslU (heat shock stress response), ATP synthase subunit alpha (mitochondrial, ATP catabolic process), phosphoglycerate kinase phosphoprotein, S-adenosylmethionine synthase 2 (one-carbon metabolism), pyruvate kinase (carbohydrate metabolism), chaperone protein DnaK 5, (stress response), and acetyl-CoA acetyltransferase.

Acetyl-CoA acetyltransferase plays a major role in ketone body metabolism as a mitochondrial enzyme involved in both amino acid degradation and fatty acid oxidation. It catalyzes the last step in breaking down isoleucine by converting 2-methyl-acetoacetyl-CoA into propionyl-CoA and acetyl-CoA. During ketolysis, this enzyme converts an acetoacetyl-CoA into two molecules of acetyl-CoA. Note that there is a cytosolic form of the enzyme (ACAT2) that can link two molecules of acetyl-CoA to acetoacetyl-CoA as an early step in ketogenesis and fatty acid biogenesis. We did not observe any suppression of the expression of this protein (reduced $>100$-fold) at $60 \mathrm{~min}$.

\section{0-min stress}

At 120 min under the stress caused by the presence of TcPr-10, the fungus began the process of DNA replication/repair and the synthesis of new mRNA. We observed the activation of the pentose phosphate pathway to produce ribose and the repression of the fatty acid degradation pathway. At this time, the most highly induced ( $>100$-fold) proteins were ubiquitin-conjugating enzyme (post-replication repair) and transaldolase of the pentose phosphate shunt (stress response).

Ubiquitin-conjugating enzymes, also known as E2 enzymes and more rarely as $u b i q$ uitin-carrier enzymes, perform the second step in the ubiquitination reaction that targets a pro- 
tein for degradation via proteasome. The ubiquitination process covalently attaches ubiquitin, a small protein of 76 amino acids, to a lysine residue on the target protein. Once a protein has been tagged with a single ubiquitin molecule, subsequent rounds of ubiquitination form a polyubiquitin chain that is recognized by the $19 \mathrm{~S}$ regulatory subunit of the proteasome, triggering the ATP-dependent unfolding of the target protein that allows its passage into the $20 \mathrm{~S}$ core subunit of the proteasome, where the active sites of the protease degrade the target into short peptide fragments for recycling by the cell (Moudry et al., 2012). Transaldolase of the pentose phosphate shunt (stress response) is an enzyme (EC 2.2.1.2) of the non-oxidative phase of the pentose phosphate pathway (Trujillo et al., 2013).

\section{Other proteins and functional analysis}

At 30 and 60 min after treatment with TcPR-10, we identified two proteins related to the process of autophagy, GTP-binding nuclear protein and autophagy-related protein. Autophagy is the process by which intracellular compounds are transported to the lysosome and vacuoles for cellular degradation, and the subunits generated after degradation are then reused by the cell, thereby establishing a feedback mechanism that allows the maintenance of homeostasis (Yorimitsu et al., 2007). ROS are stress-inducing signaling molecules that are produced during autophagy (Scherz-Shouval et al., 2007); therefore, the action of the TcPR-10 protein on M. perniciosa may be related to the expression of the GTP-binding nuclear protein and autophagy-related protein in all treatments in an attempt to detoxify the cell. According to Pozuelo-Rubio (2010), autophagy is modulated by 14-3-3 proteins, which exhibited the highest expression in the control treatment $(0 \mathrm{~h})$ and decreased expression over time. Conversely, the highest expression levels of autophagy markers were observed at $60 \mathrm{~min}$.

Among the proteins expressed by the fungus in response to TcPR-10-induced stress were proteins involved in the maintenance of fungal wall integrity against the oxidative damage caused by the antifungal protein. The alpha- 1 subunit of the $26 \mathrm{~S}$ proteasome is an ATPdependent protease that prevents the accumulation of degraded proteins (Vierstra, 2009) and therefore provides greater resistance to oxidative stress. However, it is possible that the observed decrease in the expression of this subunit over time correlated with an increase in the amount of oxidized proteins.

The proteins in the largest class that responded to TcPR-10 exposure were involved in several mechanisms (Figure 3). Our functional analysis showed that the majority of differentially expressed proteins identified by MS were involved in stress response (29\%) and cell metabolism (22\%), indicating a fungal response to maintain homeostasis and suggesting possible mechanisms of action of TcPR-10 upon and after contact.

Several PR-10 proteins with antifungal activities have been described in the literature, for example, maize PR-10 proteins (Xie et al., 2010) and JcPR-10a (Agarwal et al., 2013). Pungartnik et al. (2009) investigated mutants of $S$. cerevisiae DNA repair, membrane transport, metal transport, and antioxidant defense genes to elucidate the ribonucleic and antifungal activities of TcPR-10 against $M$. perniciosa and $S$. cerevisiae. This study showed that TcPR10 activities involving synthesis, modification or degradation may confer multiple biological functions to individual genes. The involved proteins were related to biosynthesis, carbohydrate metabolism, assembly, folding, translational regulation, and amino acid biosynthesis and transport. These modifications and their impacts on virulence, intracellular signal cascades and other biological processes represent potential areas for further investigation. 


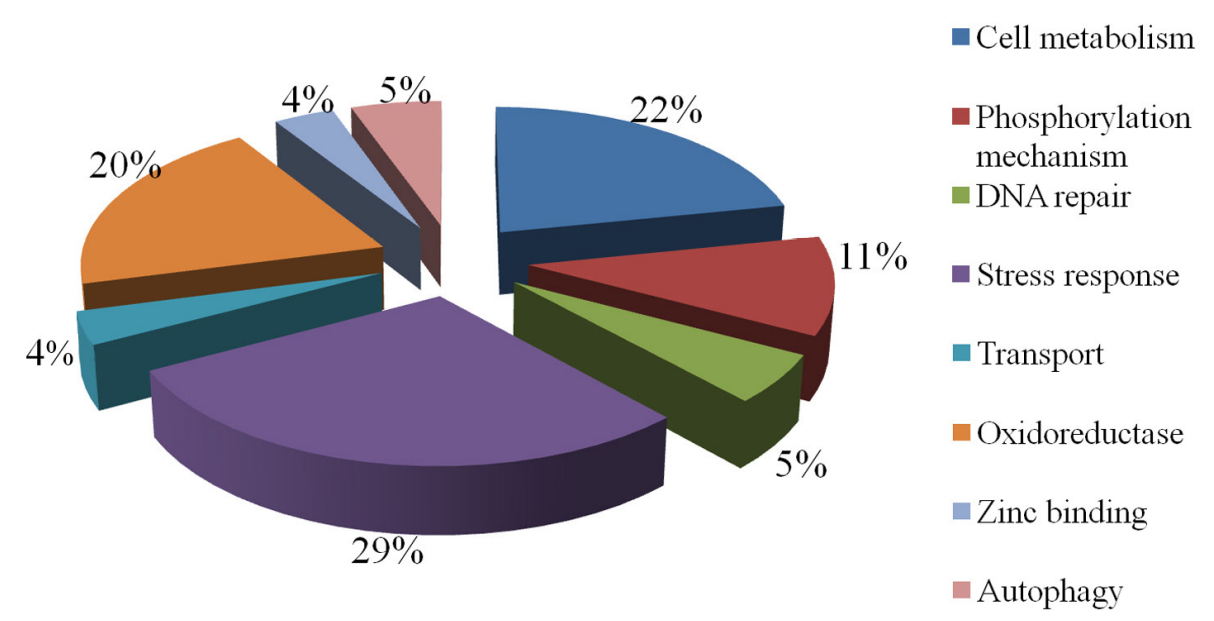

Figure 3. Representation of the distribution of differentially expressed proteins identified in Moniliophthora perniciosa according to biological process. Categorizations were based on information provided by the online resource UniProt classification system.

We observed that TcPR-10 induced interesting metabolic responses in the fungus at all times analyzed. There was a large increase in the abundance $(\% \mathrm{vol})$ of several proteins, with values above 100X compared with the control treatment. TcPR-10 has a strong ribonuclease action that induces a reaction in the fungus $M$. perniciosa in the initial attempt to eliminate the toxic effects of this antifungal compound. Thus, at the 30 - and 60 -min time points, corresponding to the initial period of stress, the major induced proteins are related to the mobilization of ATP and the activation of proteins with antioxidant action. At $120 \mathrm{~min}$, the cells begin the process of repairing the damage caused by TcPR-10, which is degraded over time, activating the pentose phosphate pathway and repressing the fatty acid degradation process. Globally, the proteins identified in this analysis are activated to detoxify the cell and to generate energy to ensure its survival. Thus, we observed that TcPR-10 can overcome the resistance of the fungus, acting as an effective antifungal agent for the periods tested.

In summary, this study describes the first proteomic analysis of the phytopathogenic fungus M. perniciosa in response to the TcPR-10 protein of cocoa. The identification of proteins that are differentially expressed after treatment with TcPR-10 provides new information that may be used to unravel the molecular basis of the pathogenesis of these fungi in the presence of antifungal molecules. The findings in this study, namely of the expression of proteins involved in DNA repair, zinc binding and the ROS response and, in particular, stress response proteins, together demonstrate the great potential for TcPR10 to be used as an antifungal agent against $M$. perniciosa. Further research is required to analyze the behavior of the differentially expressed proteins at other time points and to identify additional proteins involved in the TcPR-10 response.

\section{ACKNOWLEDGMENTS}

Research supported by Conselho Nacional de Desenvolvimento Científico e Tecnológico (CNPq, Brazil) and Fundação de Amparo à Pesquisa da Bahia (FAPESB, Brazil). F.A.C. 
Silva was supported by CAPES. The authors are thankful to Samuel Saito, PhD, Julian Santana, Msc., and Leila Lopes for their help in the identification of proteins by mass spectrometry.

\section{REFERENCES}

Agarwal P, Bhatt V, Singh R, Das M, et al. (2013). Pathogenesis-related gene, JcPR-10a from Jatropha curcas exhibit RNase and antifungal activity. Mol. Biotechnol. 54: 412-425.

Aime MC and Phillips-Mora W (2005). The causal agents of witches' broom and frosty pod rot of cacao (chocolate, Theobroma cacao) form a new lineage of Marasmiaceae. Mycologia 97: 1012-1022.

Bantignies B, Séguin J, Muzac I, Dédaldéchamp F, et al. (2000). Direct evidence for ribonucleolytic activity of a PR-10like protein from white lupin roots. Plant Mol. Biol. 42: 871-881.

Cagas SE, Jain MR, Li H and Perlin DS (2011). Profiling the Aspergillus fumigatus proteome in response to caspofungin. Antimicrob. Agents Chemother. 55: 146-154.

Filho DF, Pungartnik C, Cascardo JC and Brendel M (2006). Broken hyphae of the basidiomycete Crinipellis perniciosa allow quantitative assay of toxicity. Curr. Microbiol. 52: 407-412.

Flores T, Alape-Girón A, Flores-Díaz M and Flores HE (2002). Ocatin. A novel tuber storage protein from the andean tuber crop oca with antibacterial and antifungal activities. Plant Physiol. 128: 1291-1302.

Fu H, Subramanian RR and Masters SC (2000). 14-3-3 proteins: structure, function, and regulation. Annu. Rev. Pharmacol. Toxicol. 40: 617-647.

Gesteira AS, Micheli F, Carels N, Da Silva AC, et al. (2007). Comparative analysis of expressed genes from cacao meristems infected by Moniliophthora perniciosa. Ann. Bot. 100: 129-140.

Hara MR, Agrawal N, Kim SF, Cascio MB, et al. (2005). S-nitrosylated GAPDH initiates apoptotic cell death by nuclear translocation following Siah1 binding. Nat. Cell Biol. 7: 665-674.

He M, Xu Y, Cao J, Zhu Z, et al. (2013). Subcellular localization and functional analyses of a PR10 protein gene from Vitis pseudoreticulata in response to Plasmopara viticola infection. Protoplasma 250: 129-140.

Heller J and Tudzynski P (2011). Reactive oxygen species in phytopathogenic fungi: signaling, development, and disease. Annu. Rev. Phytopathol. 49: 369-390.

Huang WC and Tang IC (2007). Bacterial and Yeast Cultures-Process Characteristics, Products, and Applications. Bioprocessing for Value-Added Products from Renewable Resources: New Technologies and Applications, Dublin, 185-223.

Islam MA, Sturrock RN, Holmes TA and Ekramoddoullah AK (2009). Ultrastructural studies of Phellinus sulphurascens infection of Douglas-fir roots and immunolocalization of host pathogenesis-related proteins. Mycol. Res. 113: 700-712.

Izawa S, Maeda K, Miki T, Mano J, et al. (1998). Importance of glucose-6-phosphate dehydrogenase in the adaptive response to hydrogen peroxide in Saccharomyces cerevisiae. Biochem. J. 330 (Pt 2): 811-817.

Kilaru A and Hasenstein KH (2005). Development and pathogenicity of the fungus Crinipellis perniciosa on interaction with cacao leaves. Phytopathology 95: 101-107.

Kim SG, Kim ST, Wang Y, Yu S, et al. (2011). The RNase activity of rice probenazole-induced protein1 (PBZ1) plays a key role in cell death in plants. Mol. Cells 31: 25-31.

Liu JJ, Ekramoddoullah AK, Piggott N and Zamani A (2005). Molecular cloning of a pathogen/wound-inducible PR10 promoter from Pinus monticola and characterization in transgenic Arabidopsis plants. Planta 221: 159-169.

Lytle BL, Song J, de la Cruz NB, Peterson FC, et al. (2009). Structures of two Arabidopsis thaliana major latex proteins represent novel helix-grip folds. Proteins 76: 237-243.

Menezes SP, dos Santos JL, Cardoso TH, Pirovani CP, et al. (2012). Evaluation of the allergenicity potential of TcPR-10 protein from Theobroma cacao. PLoS One 7: e37969.

Meyer Y, Grosset J, Chartier Y and Cleyet-Marel JC (1988). Preparation by two-dimensional electrophoresis of proteins for antibody production: antibodies against proteins whose synthesis is reduced by auxin in tobacco mesophyll protoplasts. Electrophoresis 9: 704-712.

Moudry P, Lukas C, Macurek L, Hanzlikova H, et al. (2012). Ubiquitin-activating enzyme UBA1 is required for cellular response to DNA damage. Cell Cycle 11: 1573-1582.

Mur LA, Sturgess FJ, Farrell GG and Draper J (2004). The AoPR10 promoter and certain endogenous PR10 genes respond to oxidative signals in Arabidopsis. Mol. Plant Pathol. 5: 435-451.

Nevoigt E and Stahl U (1997). Osmoregulation and glycerol metabolism in the yeast Saccharomyces cerevisiae. FEMS Microbiol. Rev. 21: 231-241.

Park CJ, Kim KJ, Shin R, Park JM, et al. (2004). Pathogenesis-related protein 10 isolated from hot pepper functions as a ribonuclease in an antiviral pathway. Plant J. 37: 186-198. 
Pinto MP and Ricardo CP (1995). Lupinus albus L. pathogenesis-related proteins that show similarity to PR-10 proteins. Plant Physiol. 109: 1345-1351.

Pirovani CP, Carvalho HA, Machado RC, Gomes DS, et al. (2008). Protein extraction for proteome analysis from cacao leaves and meristems, organs infected by Moniliophthora perniciosa, the causal agent of the witches' broom disease. Electrophoresis 29: 2391-2401.

Pozuelo-Rubio M (2011). Regulation of autophagic activity by 14-3-3zeta proteins associated with class III phosphatidylinositol-3-kinase. Cell Death Differ. 18: 479-492.

Pungartnik C, da Silva AC, de Melo SA, Gramacho KP, et al. (2009). High-affinity copper transport and Snq2 export permease of Saccharomyces cerevisiae modulate cytotoxicity of PR-10 from Theobroma cacao. Mol. Plant Microbe Interact. 22: 39-51.

Ronne H (1995). Glucose repression in fungi. Trends Genet. 11: 12-17.

Scarpari LM, Meinhardt LW, Mazzafera P, Pomella AW, et al. (2005). Biochemical changes during the development of witches' broom: the most important disease of cocoa in Brazil caused by Crinipellis perniciosa. J. Exp. Bot. 56: 865-877.

Scherz-Shouval R, Shvets E, Fass E, Shorer H, et al. (2007). Reactive oxygen species are essential for autophagy and specifically regulate the activity of Atg4. EMBO J. 26: 1749-1760.

Thompson DM, Lu C, Green PJ and Parker R (2008). tRNA cleavage is a conserved response to oxidative stress in eukaryotes. RNA 14: 2095-2103.

Trujillo P, Ketchum S, McLain K and Sommerville L (2013). NMR Metabolite Profiling of Acidobacterium capsulatum Grown in Minimal Media with Glucose as the Sole Carbon Source. In: 8th Annual Natural \& Behavioral Sciences Undergraduate Research Symposium Program, Durango.

Vierstra RD (2009). The ubiquitin-26S proteasome system at the nexus of plant biology. Nat. Rev. Mol. Cell Biol. 10: 385-397.

Wandinger SK, Richter K and Buchner J (2008). The Hsp90 chaperone machinery. J. Biol. Chem. 283: 18473-18477.

Xie YR, Chen ZY, Brown RL and Bhatnagar D (2010). Expression and functional characterization of two pathogenesisrelated protein 10 genes from Zea mays. J. Plant Physiol. 167: 121-130.

$\mathrm{Xu} \mathrm{F}$ (2005). Applications of oxidoreductases: recent progress. Ind. Biotechnol. 1: 38-50.

Yan Q, Qi X, Jiang Z, Yang S, et al. (2008). Characterization of a pathogenesis-related class 10 protein (PR-10) from Astragalus mongholicus with ribonuclease activity. Plant Physiol. Biochem. 46: 93-99.

Yorimitsu T, Zaman S, Broach JR and Klionsky DJ (2007). Protein kinase A and Sch9 cooperatively regulate induction of autophagy in Saccharomyces cerevisiae. Mol. Biol. Cell 18: 4180-4189.

Zhao H, Ruberu K, Li H and Garner B (2013). Analysis of subcellular $\left[{ }^{57} \mathrm{Co}\right]$ cobalamin distribution in SH-SY5Y neurons and brain tissue. J. Neurosci. Methods 217: 67-74. 